\title{
Erratum to: Effects of Chronic Dust Load On Leaf Pigments of the Landscape Plant Murraya Paniculata
}

\author{
Kamran Shah ${ }^{1,2} \cdot$ Noor ul Amin $^{2} \cdot$ Imran Ahmad ${ }^{2} \cdot$ Gulshan Ara $^{3} \cdot$ Xiaolin Ren $^{1} \cdot$ Libo Xing $^{1}$
}

Published online: 7 October 2019

๑) Springer-Verlag GmbH Deutschland, ein Teil von Springer Nature 2019

\section{Erratum to:}

\section{Gesunde Pflanzen 2019}

https://doi.org/10.1007/s10343-019-00469-3

Unfortunately there was a technical problem with the conversion of the formulas in the PDF file of this article. The hyphen between chlorophyll-a and chlorophyll-b, as well as between pheophytin-a and pheophytin-b was not inserted in the following formulas:

Chlorophyll-a $(\mu \mathrm{g} / \mathrm{ml})=12.25 A_{663.6}-2.55 A_{646.6}$

Chlorophyll-b $(\mu \mathrm{g} / \mathrm{ml})=20.31 A_{646.6}-4.91 A_{663.6}$

Total chlorophyll $(\mu \mathrm{g} / \mathrm{ml})=17.76 A_{646.6}+7.34 A_{663.6}$

Carotenoids $(\mu \mathrm{g} / \mathrm{ml})=4.69 A_{440.5}-0.267 \mathrm{Chl}-\mathrm{a}+$ Chl-b
Protoporphyrin $(\mathrm{nmol})=196.25 A_{575}-46.6 A_{590}-58.68 A_{628}$ Magnesium Protoporphyrin (nmol)

$=61.81 A_{590}-23.77 A_{575}-3.55 A_{628}$

Protochlorophyllide $(\mathrm{nmol})=42.59 A_{628}-34.22 A_{575}$

$$
-7.25 A_{590}
$$

Chlorophyllide-a $(\mathrm{mmol})=A_{667} / 74.9$

Chlorophyllide-b $(\mathrm{mmol})=A_{650} / 47.2$

Polar Carotenoid $(\mu \mathrm{g} / \mathrm{ml})=4.69 A_{440.5}-0.267 \mathrm{TChl}$

Pheophytin-a $(\mu \mathrm{g} / \mathrm{ml})=22.42 A_{665.4}-6.81 A_{653.4}$

Pheophytin-b $(\mu \mathrm{g} / \mathrm{ml})=40.17 A_{653.4}-18.58 A_{665.4}$

Non polar Carotenoids $(\mu \mathrm{g} / \mathrm{ml})=\frac{1000 A_{470}-4.78 A_{653.4}}{164}$

The original article has been corrected.

The online version of the original article can be found under https://doi.org/10.1007/s10343-019-00469-3.

Xiaolin Ren

renxl@nwsuaf.edu.cn

$\triangle \quad$ Libo Xing

libo_xing@nwsuaf.edu.cn

1 College of Horticulture, Northwest Agriculture \& Forestry University, Yangling, Shaanxi, China

2 Department of Horticulture, University of Agriculture, Peshawar, Pakistan

3 Institute of Biotechnology and Genetic Engineering, University of Agriculture, Peshawar, Pakistan 